\title{
Selfconsistent Electron Beam Charging of Non-Conductive Specimen in SEM and ESEM
}

\author{
H.-J. Fitting ${ }^{*}$ and M. Touzin ${ }^{* *}$ \\ *Physics Department, University of Rostock, Universitaetsplatz 3, D 18051 Rostock, Germany \\ ${ }^{* *}$ Lab. des Surfaces, Ecole Centrale de Lyon, 36 ave. Guy de Collongue, F-69134 Ecully, France
}

The aim of the present work is to simulate the electron beam charge injection and the associated selfconsistent charge transport in bulk and insulating specimen. The new flight-drift model (FDM), [1], of selfconsistent electron transport and electrical charge storage in wide-gap insulators reflects a more realistic simulation of these processes in dielectric and insulating materials than the former mainly ballistic model [2]. So electron-hole creation, their ballistic flight, followed by diffusion and/or field-drift transport, and finally trapping in localized states and electron-hole recombination take place [1]. Thus a total resulting current $j(x)$ is obtained:

$$
j(\mathrm{x}, \mathrm{t})=-\boldsymbol{j}_{\mathrm{PE}}(\mathrm{x}, \mathrm{t})-\boldsymbol{j}_{\mathrm{SE}}(\mathrm{x}, \mathrm{t})+\boldsymbol{j}_{\mathrm{HB}}(\mathrm{x}, \mathrm{t})-\boldsymbol{j}_{\mathrm{ED}}(\mathrm{x}, \mathrm{t})+\boldsymbol{j}_{\mathrm{HD}}(\mathrm{x}, \mathrm{t})
$$

where PE indicates the injected primary electrons, SE the ballistic secondary electrons, HB the ballistic holes and ED and HD the diffusing and drifting electrons and holes, respectively. The latter ones can recombine or are captured in shallow and deep traps, as well as released by the field and temperature-dependent Poole-Frenkel effect. Thus a selfconsistent charge transport is obtained with a strong feed-back of the stored (trapped) charges and their electric field to the subsequent currents in eq. (1). The experimentally accessable quantities of field assisted secondary electron emission $\sigma$ as well as the resulting surface potential $\mathrm{V}_{0}$ due to internal current $j(\mathrm{x}, \mathrm{t})$, charge $\rho(\mathrm{x}, \mathrm{t})$, field $F(\mathrm{x}, \mathrm{t})$, and potential $V(\mathrm{x}, \mathrm{t})$ distributions are obtained, see Fig.1, and in fair agreement with measurements of secondary electron emission rates $\sigma\left(E_{0}, d\right)$, [3], and surface potentials $V_{0}\left(E_{0}, d\right)$, [4].

A: The charging of open, i.e. non-covered and floating insulating bulk samples is strongly controlled by the surface potential $\mathrm{V}_{0}(x=0)$ and the consequent electron beam retarding for negative charging $\mathrm{V}_{0}<0$ or even beam accelleration for positive charging $\mathrm{V}_{0}>0$ according to the affection of the initial energy $E_{0}^{\prime}=E_{0}+e V_{0}$. Thus the maximum range of incident primary electrons is rapidly diminished for high beam energies $\mathrm{E}_{0}>5 \mathrm{keV}$ by negative charging and the internal current, charge and field distributions are shrinked strongly towards the surface.

B: Contrary to open samples the positive-ion-covered samples of the ESEM techniques possess an only slightly negative surface potential fixed by the positive ions at about $\mathrm{V}_{0}=-0.5 \mathrm{kV}$. Here the electron excitation is almost not affected by beam retarding and the charge and field distributions, see Fig.2, show less fluctuations, however, an increase in magnitude towards the surface.

C: A similar effect is given in conventional metal or conducting material coated insulating samples. Usually the coating layer is grounded and no electron beam retarding field effects are observed, also within sample where the negative potential distribution does not exceed several tens of Volt, i.e. $V(x)<-50$ Volt. Thus the exciting electron beam is not affected neither in the front of the surface in vacuum nor in the internal bulk insulator. However, one cannot neglect the additional scattering of the incident electron beam as well as of backscattered and secondary electrons within the coating layer. Thus, in order to prevent electrical charging of insulating samples, the positive-ion-covered surface bears certain advantages versus metal coating, more details see in [1]. 


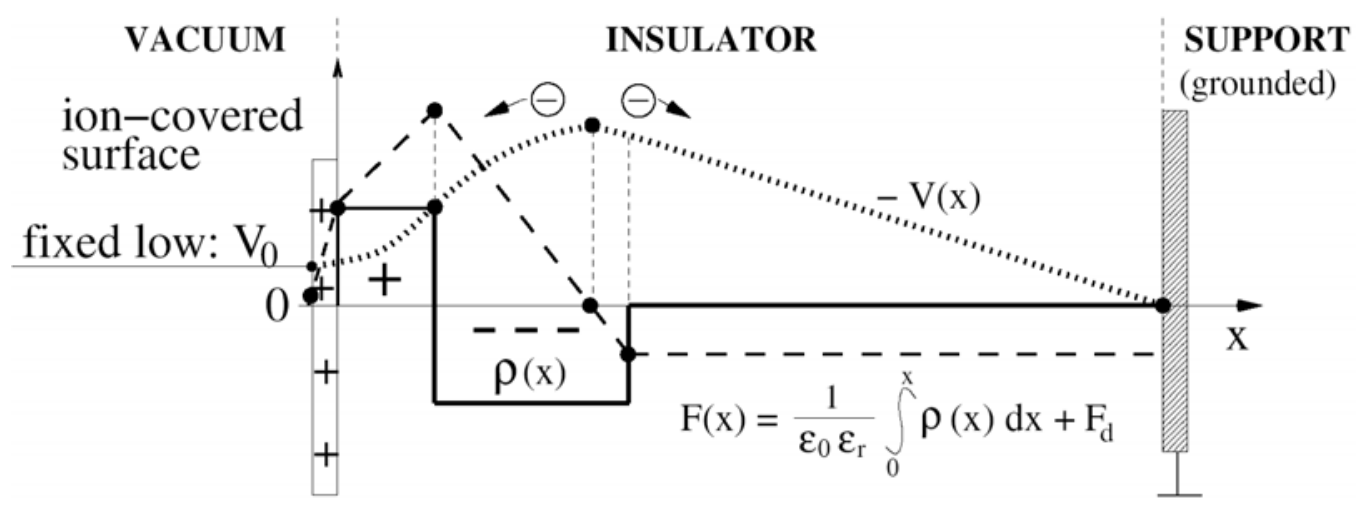

Fig. 1. A schematic plus-minus distribution $\rho(\mathrm{x})$ beneath the positive ion-covered surface of an insulating target in ESEM and the respective field $F(\mathrm{x})$ and potential $V(\mathrm{x})$ distributions.

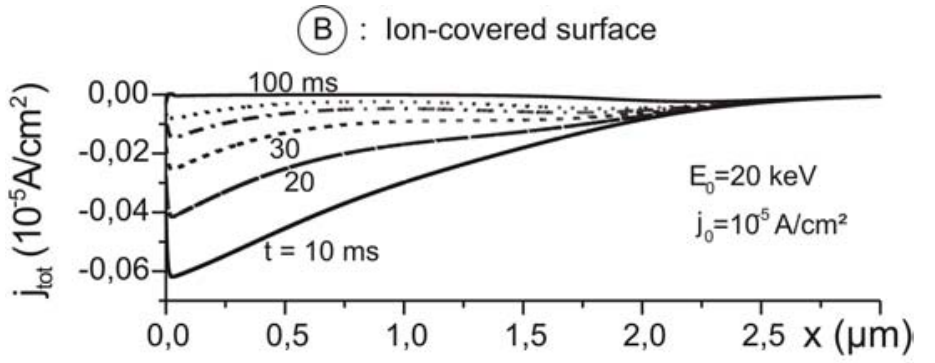

Fig. 2. Current $j_{\text {tot }}(\mathrm{x})$, charge $\rho(\mathrm{x})$, electric field $F(\mathrm{x})$, and potential $V(\mathrm{x})$ depth distributions beneath the positive ion-covered surface ( $V_{0}=-500$ Volt) of a $3 \mathrm{~mm}$ alumina target in dependence on irradiation

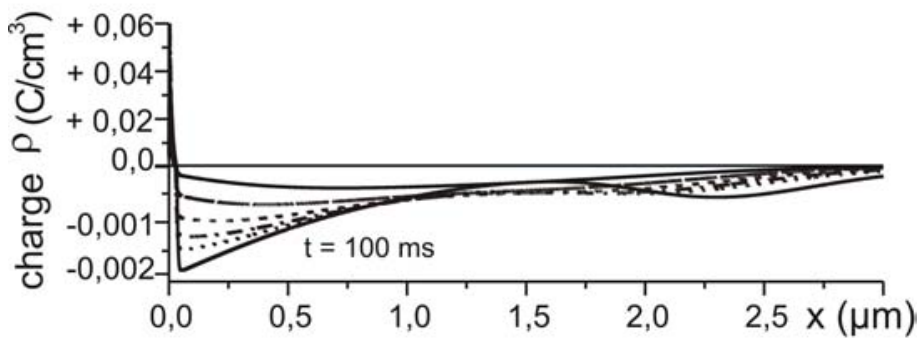
time $t$; incident electron beam energy: $E_{0}=20 \mathrm{keV}$ and current density: $j_{0}=10^{-5} \mathrm{~A} / \mathrm{cm}^{2}$.

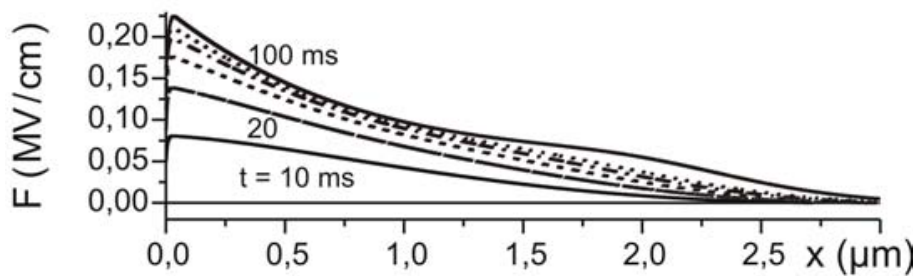

\section{References}

[1] M. Touzin, D. Goeuriot, C. Guerret-Piécourt, D. Juvé, D.

Treheux, H.-J. Fitting, J. Appl.

Physics (2006) submitted.

[2] X. Meyza, D. Goeuriot, C. Guerret-Piecourt, D. Treheux, H.-

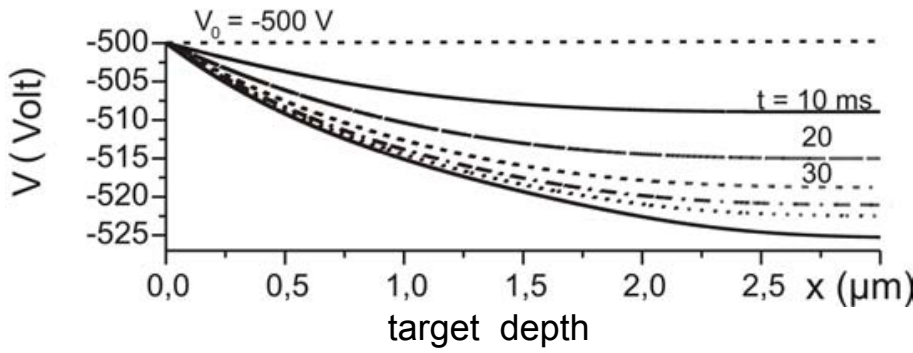
J. Fitting, J. Appl. Physics 94 (2003) 5384-5392.

[3] H.-J. Fitting, D. Hecht, Physica Status Solidi (a) 108 (1988) 265-273. [4] H.-J. Fitting, P. Magdanz, W. Mehnert, D. Hecht, Th. Hingst, Physica Status Solidi (a) 122 (1990) 297-309. 